\title{
Laparoscopic Upper-Pole Nephroureterectomy in Infants
}

\author{
Marcio L. Miranda, Antonio G. Oliveira-Filho, Patricia T. Carvalho, Elaine Ungersbock, \\ Hugo Olimpio, Joaquim M. Bustorff-Silva
}

Division of Genitourinary Surgery, Pediatric Surgery Unit, State University of Campinas, Unicamp, Campinas, Sao Paulo, Brazil

\begin{abstract}
Objective: Report the results of laparoscopic upper-pole nephroureterectomy in infants.

Materials and Methods: Six consecutive infants underwent 7 laparoscopic upper-pole nephroureterectomy. Pre and postoperative evaluation included renal sonography, voiding cystourethrogram and renal scintigraphy. All infants showed upper-pole exclusion. Surgery was performed through a transperitoneal approach with full flank position in all infants. Three or 4 ports were used according to the necessity of retracting the liver. The distal ureter was ligated close to the bladder whenever reflux was present and the dysplastic upper-pole was divided with the help of an electrocautery. Data regarding operative time, postoperative use of analgesics, time to resume oral feeding, hospital stay and tubular function were collected and analyzed.

Results: All procedures were concluded as planned. Mean operative time was $135 \mathrm{~min}$. One patient underwent staged bilateral upper-pole nephrectomy. There were no complications and the postoperative hospital stay was 48 hours in 5 procedures and 24 hours in 2 procedures. Pain medication was required only in the first day. Renal tubular function showed improvement in half of the cases.

Conclusion: Laparoscopic partial nephrectomy is a safe and feasible procedure in infants. Due to the magnification provided by the lenses, a better vision of the structures is achieved, facilitating selective dissection of vascular upper-pole, renal parenchyma and distal ureter. This approach is less damaging to the lower pole, and is associated to low morbidity and a short hospital stay.
\end{abstract}

Key words: laparoscopy; infants; nephrectomy

Int Braz. J Urol. 2007; 33: 87-93

\section{INTRODUCTION}

In pediatric practice, the use of minimally invasive surgery is on the rise due to its innumerous advantages over open surgery (1-3). Nephrectomy, which was one of the first laparoscopic procedures performed in children, has gained significant acceptance, especially due to the minimal morbidity, shorter hospital stay and improved cosmesis
$(1,2,4,5)$. Since the first report by Jordan and Winslow in 1993, the laparoscopic approach has become the procedure of choice for heminephrectomy $(6,7)$. The retroperitoneal approach was proposed by GILL et al. in 1994 (8), but its use was restricted in infants due to the high incidence of peritoneal perforation (9). The purpose of this study is to report the results of a consecutive series of laparoscopic upper-pole nephroureterectomy proce- 
dures, with special emphasis in the function of the remaining kidney.

\section{MATERIALS AND METHODS}

Seven upper-pole nephroureterectomies were performed in six infants between January 2002 and January 2005 . Clinical data were obtained by chart review. Age at operation ranged from 5 to 20 months (median: 9.5 months). In the case of a boy with bilateral duplex system, a second procedure was done 5 months after the first surgery. All infants (except one with recurrent urinary tract infection) had a prenatal diagnosis of pyeloureteral duplex system. This diagnosis was confirmed by ultrasonography, voiding cystourethrogram and scintigraphy after birth. The ${ }^{99 m}$ Tc-DMSA scintigraphy revealed duplicity of the renal unit with upper pole exclusion in all cases. Cystogram showed ureterocele in 1 case and one child had vesicoureteral reflux in both units.

The procedure was done as described by Desgrandchamps et al. 1999 (10). The transperitoneal approach was achieved with the patient in the lateral decubitus position with the operative side up and the lumbar region slightly flexed (Figure-1). The peritoneum was insufflated with $\mathrm{CO}_{2}$ (pressure $12 \mathrm{mmHg}$ ). Three trocars were introduced (two $5 \mathrm{~mm}$ and one 10 $\mathrm{mm})$. A fourth trocar $(2 \mathrm{~mm})$ was used in case a liver retraction was needed ( 3 cases). After incising along the Toldt's line, the colon was retracted medially and the Gerota fascia was opened. Careful dissection of the ureter of the upper pole avoiding mobilization of ureter of the lower unit was done followed by the transposition of the duplicate ureter over the renal vascular pedicle. The vascular supply of the upperpole was dissected and ligated with clips and the dysplastic parenchyma was transected with electrocautery, avoiding damage to the lower half of the kidney and to its vascular pedicle. No attempt was made to suture the renal capsule over the open parenchymal surface. Finally, distal ureter was either clipped close to the bladder if reflux was present, or emptied and left opened whenever an ureterocele was present. The retroperitoneal space was sutured and the incisions were infiltrated with bupivacain. The operative time,

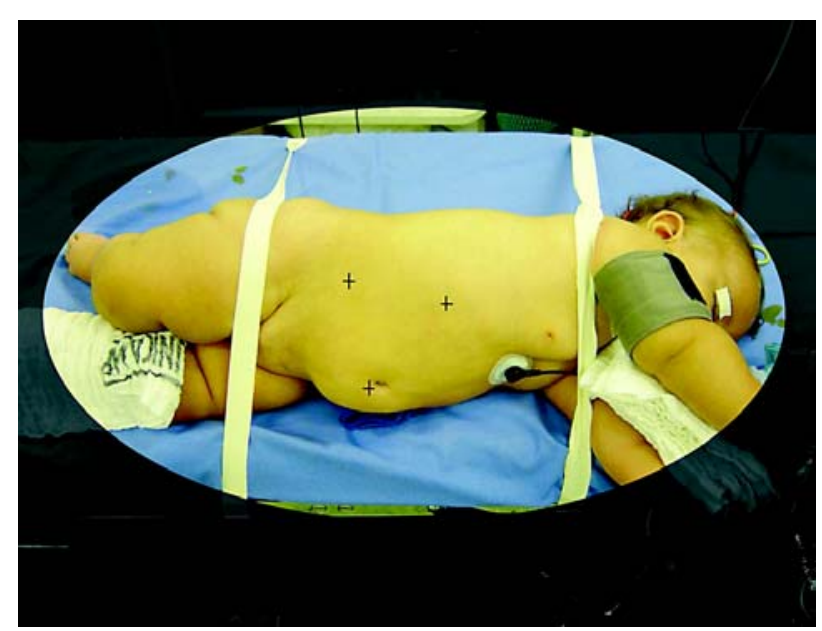

Figure 1 - Photographic representation of a patient in lateral decubitus showing the location of the trocars.

need for analgesics, time to resume oral feeding and length of hospital stay were assessed. Scintigraphic study to access the tubular function was done usually six months after the surgery.

\section{RESULTS}

All the procedures were completed laparoscopically with a mean operative time of 135 minutes (range: 120 to 160 minutes). The estimated blood loss was minimal and no major per-operative complications were observed. Five infants were fed 4 hours after returning to their beds and the remaining in the day following the surgery. The length of hospital stay was 48 hours for 5 infants and 24 hours for the other 2. Pain medication was required only in the first postoperative day. The histopathological results indicated the presence of renal dysplasia in 3 specimens and chronic pyelonephritis in 4 .

Mean follow-up was 18 months. ${ }^{99 \mathrm{~m}} \mathrm{Tc}-\mathrm{DMSA}$ scintigraphy showed an improvement or maintenance of tubular function in all infants (Figure-2).

\section{COMMENTS}

Because the clinical diagnosis of duplex kidney is presumptive and renal dysplasia of the upper 


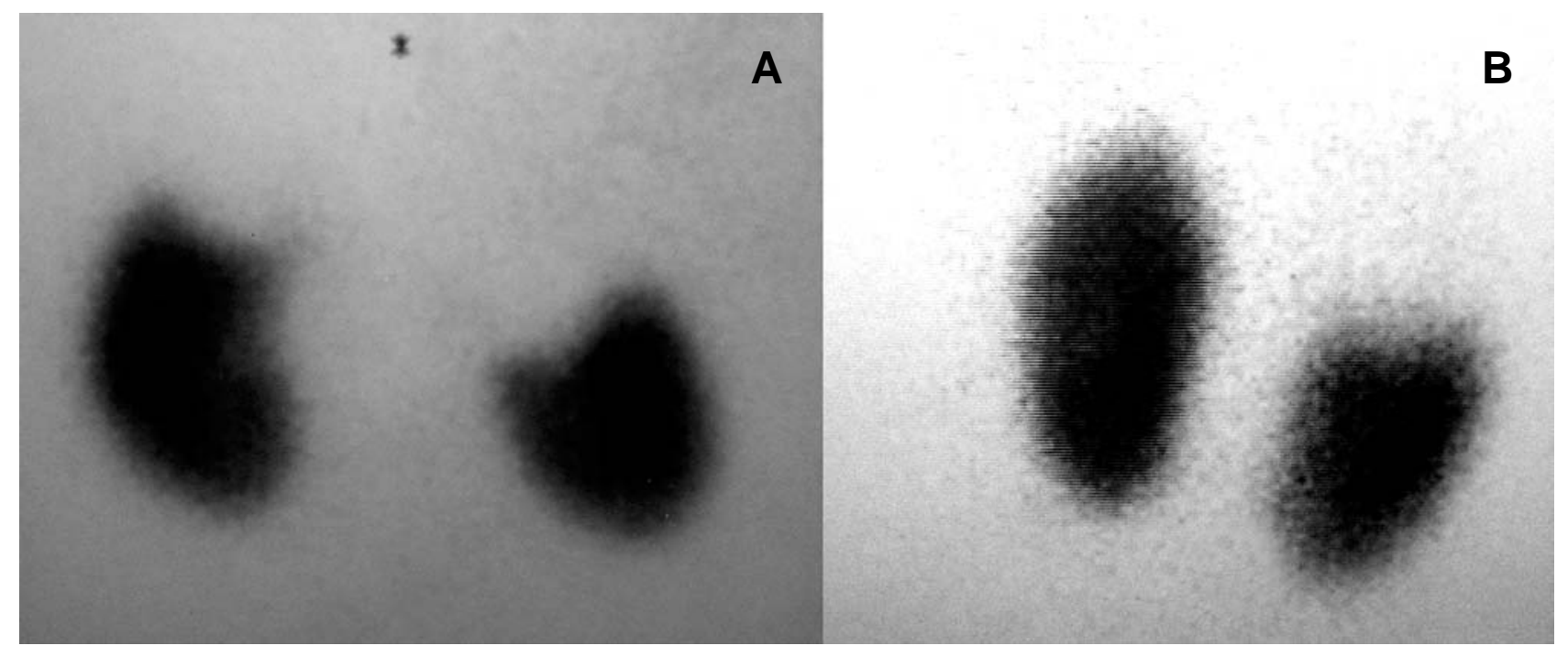

Figure 2 - Selected IMAGES of the ${ }^{99 m} T c$-DMSA showing bilateral upper-pole exclusion on the preoperative (A) and parenchymal accommodation on the postoperative period $(B)$.

pole may remain asymptomatic, prenatal or incidental sonographic findings have contributed to early diagnosis (11). In this series, 6 out of 7 infants had suspected prenatal diagnosis of duplex system and were therefore referred early to the specialist.

Several surgical approaches to nephrectomy of the upper pole have been described. The classic dorsal lumbotomy approach ensures a great exposure but requires a large incision, intense renal mobilization and is associated to the possibility of atrophy of the remaining kidney $(1,5,12)$. Jednak et al. (2000) described a rapid, safe and easy technique of open heminephrectomy through supracostal approach, which however had to incise parietal muscles and diaphragm to gain greater exposure (13). With the advances and development of appropriated instruments for children, improvement of the techniques with resulting lower rates of morbidity, minimally invasive surgery is becoming common in the pediatric surgery $(1,3)$. An important contribution of video surgery in partial nephrectomy is that the perfect view of the pedicle of both units and delimitation after vascular clamping allows orderly sectioning of the parenchyma, avoiding damage to the intact remaining unit $(5,12)$. Some authors recommend the use of a harmonic scalpel or argon beam coagulator to resect the parenchyma; however, when the vascular delimitation is clear, this section does not represent a problem $(1,12)$. There is still some controversy regarding the choice of either the transperitoneal or retroperitoneal approach. Supporters of the retroperitoneal approach believe that it provides exposure of the posterior aspect of the kidney units, avoiding dissection of the kidney pedicle, which can be preserved $(5,14)$. This approach may be posterior or lateral. Borzi et al. have compared these two approaches and concluded that the posterior approach is preferable for nephrectomies that do not need ureterectomy (9). The lateral approach, on the other hand, provides better access for complete resection of the ureter.

The main inconvenience of the retroperitoneal access is the higher incidence of peritoneal tears in infants, which prevent the creation of an adequate retropneumoperitoneum. This is the most common complication and also the main cause of conversion to open surgery $(9,12)$. In some cases, peritoneal microperforations and consequent ventilatory changes may occur. On the contrary, besides avoiding theses complications, the transperitoneal approach also offers an excellent approach to the vascular bundle with minimum lower pole mobilization and minimal morbidity when compared to retroperitoneal approach (2). Nevertheless, there is no conclusive medical evidence 
that favors either the retro or transperitoneal approach $(3,15)$. Like others, we also use the retroperitoneal approach for children over two years of age or to perform a total nephrectomy (14).

The subjectivity of evaluating postoperative pain in children, made analysis of the data very difficult. Reduction of postoperative pain is apparent but very hard to prove in many controlled series (2).

Assessment of the postoperative tubular function has not been stressed in the literature, most probably due to the low incidence of the lesions in the remaining unit. Scintigraphic evaluation is more qualitative than quantitative. In this series, half of the cases presenting with preoperative ureterohydronephrosis and compression of the lower pole, showed recover on postoperative scintigraphy. This observation is probably due to parenchymal accommodation and not to an actual improvement of tubular function. The ${ }^{99 m} \mathrm{Tc}-D M S A$ analysis of the tubular function was considered adequate for postoperative evaluation since it demonstrated improved uptake of some renal units, justifying the use of video assisted renal surgery in our service.

Most authors did not observe any difference regarding the surgical duration of laparoscopic heminephrectomy and conventional surgery $(5,16)$. The increase in operative time reported by some is probably related to the learning curve $(3,12,16)$.

\section{CONCLUSION}

Minimally invasive approach should be considered when partial nephroureterectomy is indicated, whether through a transperitoneal or a retroperitoneal approach. Magnification makes selective upper-pole dissection safe and feasible, promotes sectioning of the distal ureter without additional incisions, minimizes surgical trauma in the lower pole with minimal morbidity, improving cosmetic results and reducing hospital stay.

\section{CONFLICT OF INTEREST}

None declared.

\section{REFERENCES}

1. Peters CA: Laparoscopic and robotic approach to genitourinary anomalies in children. Urol Clin North Am. 2004; 31: 595-605.

2. Robinson BC, Snow BW, Cartwright PC, De Vries CR, Hamilton BD, Anderson JB: Comparison of laparoscopic versus open partial nephrectomy in a pediatric series. J Urol. 2003; 169: 638-40.

3. Steyaert H, Valla JS: Minimally invasive urologic surgery in children: an overview of what can be done. Eur J Pediatr Surg. 2005; 15: 307-13.

4. Koyle MA, Woo HH, Kavoussi LR: Laparoscopic nephrectomy in the first year of life. J Pediatr Surg. 1993; 28: 693-5.

5. Valla JS, Breaud J, Carfagna L, Tursini S, Steyaert $\mathrm{H}$ : Treatment of ureterocele on duplex ureter: upper pole nephrectomy by retroperitoneoscopy in children based on a series of 24 cases. Eur Urol. 2003; 43: 426-9.

6. Jordan GH, Winslow BH: Laparoendoscopic upper pole partial nephrectomy with ureterectomy. J Urol. 1993; 150: 940-3.

7. Horowitz M, Shah SM, Ferzli G, Syad PI, Glassberg KI: Laparoscopic partial upper pole nephrectomy in infants and children. BJU Int. 2001; 87: 514-6.

8. Gill IS, Delworth MG, Munch LC: Laparoscopic retroperitoneal partial nephrectomy. J Urol. 1994; 152: 1539-42.

9. Borzi PA: A comparison of the lateral and posterior retroperitoneoscopic approach for complete and partial nephroureterectomy in children. BJU Int. 2001; 87: 517-20.

10. Desgrandchamps F, Gossot D, Jabbour ME, Meria P, Teillac P, Le Duc A: A 3 trocar technique for transperitoneal laparoscopic nephrectomy. J Urol. 1999; 161: 1530-2.

11. Hulbert WC, Rabinowitz R: Prenatal diagnosis of duplex system hydronephrosis: effect on renal salvage. Urology. 1998; 51: 23-6.

12. El-Ghoneimi A, Farhat W, Bolduc S, Bagli D, McLorie G, Khoury A: Retroperitoneal laparoscopic vs open partial nephroureterectomy in children. BJU Int. 2003; 91: 532-5.

13. Jednak R, Kryger JV, Barthold JS, Gonzalez R: A simplified technique of upper pole heminephrectomy for duplex kidney. J Urol. 2000; 164: 1326-8.

14. Borzi PA, Yeung CK: Selective approach for transperitoneal and extraperitoneal endoscopic nephrectomy in children. J Urol. 2004; 171: 814-6. 
15. Guillonneau B, Ballanger P, Lugagne PM, Valla JS, Vallancien G: Laparoscopic versus lumboscopic nephrectomy. Eur Urol. 1996; 29: 288-91.
16. Janetschek G, Seibold J, Radmayr C, Bartsch G: Laparoscopic heminephroureterectomy in pediatric patients. J Urol. 1997; 158: 1928-30.

Accepted after revision:

June 31, 2006

\author{
Correspondence address: \\ Dr. Marcio Lopes Miranda \\ R. Timburí 945 \\ Campinas, SP, 13098-301, Brazil \\ E-mail: marciomiranda@terra.com.br
}

\section{EDITORIAL COMMENT}

The authors performed upper pole nephrectomy by laparoscopy in children under two years old and achieved good results. The authors are to be congratulated for their efforts in light of the fact that few articles have been published on this subject. However, I would like to comment on some of the thoughts and conclusions made by the authors. First, despite its common usage, the term "minimally invasive procedure" is not an accurate manner to address the laparoscopic upper pole nephrectomy because except for skin incision all the following steps are the same as the open surgery. Because the laparoscopic surgery was performed intraperitonially, one could actually consider it as more invasive, since the peritoneum is not entered in the open procedure. Also, a $10 \mathrm{~mm}$ trocar is not a small instrument for such a small child. I believe laparoscopic upper pole nephrectomy is the procedure of choice in older children and has been performed on our group at this age. Moreover, I do not agree with the authors' statement that in small children a large incision is needed for open surgery. The benefits of improved cosmesis and rapid recovery remain controversial in younger children and infants, where smaller incisions and quicker recovery tend to be the role in most open procedures (1). In our department we perform in very young children, the technique described by Jednak et al. and do not isolate the vascular pedicle, which minimizes the risk of vascular damage, and lower pole ischemia $(2,3)$. This procedure is fast, the patients are discharged in 24 hours and there is no need for excessive pain medication at this age.

The authors commented that 5 children were discharged within 48 hours and that pain medication was only necessary for 24 hours in all patients. This raises the question, what were these 5 infants doing at the hospital for 24 hours longer if there was no more pain?

The authors reported that there was improvement in renal function on the operated side. However, this is not possible since according to the authors, there was no upper pole function before the operation and all upper pole tissue was removed. There was no comment about the extent of this improvement, but certainly this cannot be attributed to 
a better technique. A maximum of 5\% difference among two renal DMSA scans would be expected and this is just an artifact and therefore I do not think that renal accommodation is a good explanation.

Furthermore because there is no control group, the authors cannot justify any advantage of upper pole laparoscopy over open surgery. Prospective studies comparing different surgical approaches are warranted.

\section{REFERENCES}

1. Wallis MC, Khoury AE, Lorenzo AJ, Pippi-Salle JL, Bagli DJ, Farhat WA: Outcome analysis of retroperitoneal laparoscopic heminephrectomy in children. J Urol. 2006; 175: 2277-82.

2. Jednak R, Kryger JV, Barthold JS, Gonzalez R: A simplified technique of upper pole heminephrectomy for duplex kidney. J Urol. 2000; 164: 1326-8.

3. Barroso U Jr, Vinhaes AJ, Barros MS, Calado AA, Macedo A Jr, Srougi M: Simplified upper pole nephrectomy: initial experience. Int Braz J Urol. 2005; 31 : 157-60.

\section{Dr. Ubirajara Barroso Jr. Section of Urology Federal University of Bahia Salvador, Bahia \\ E-mail:ubarroso@uol.com.br}

\section{EDITORIAL COMMENT}

Partial nephrectomy is an uncommon procedure in children. This study, therefore, is a welcome opportunity to re-visit this problem. Indeed antenatal diagnosis has uncovered many urologic anomalies, including ureteral duplications, which are asymptomatic (6 cases in this series). Nevertheless, spontaneous resolution seems unlikely for most ectopic ureters and ureteroceles (1). So as in this series, in case of duplex system with minimal or non-functioning upper pole, heminephrectomy is recommended for the affected upper pole (2). A subtotal ureterectomy is usually sufficient, certainly when there is no associated ureterocele. Once that established rest the type of approach.

The gold standard approach still uses a classical flank incision. One of the main problems of this type of operation is that it requires a complete mobilization of the kidney and the vessels. However, vessels of babies are prone to spasm. Failure of excretion of the lower pole therefore is the main postoperative complication. Minimal invasive surgery will probably decrease this complication due to better vision of the vessels (magnification) and the fact that heminephrectomy will be done "in situ". The minimal invasive approach however may be transperitoneal or retroperitoneal (3). The transperitoneal approach, as described in authors' paper, provides a wider exposition but requires colon mobilization and unnecessary opening of the peritoneum. Complications are described (4). Patient's position may be lateral as in this series or more frequently supine with a tilted table. The retroperitoneal access is more "natural", faster in experienced hands, but requires creation of a working space. This way undoubtedly gives the best hilum's exposition. Conversion rate is higher, in part due to the learning curve and a thin peritoneum in babies (3 and authors).

Postoperative assessment of tubular function is a finding of importance in this paper. The authors 
should be encouraged to report longer term-follow up and perhaps to design a study in collaboration with their scintigraphists and nephrologists in order to better understand this phenomenon.

\section{REFERENCES}

1. Keating MA: Ureteral Duplication Anomalies: Ectopic Ureters and Ureteroceles. In: Docimo SG (ed.), Clinical Pediatric Urology. United Kingdom, Informa Healthcare. 2007, fifth edition, 593-648.
2. Smith EL, Ritchie EL, Maizels M, Zaontz MR, Hsueh W, Kaplan WE, et al.: Surgery for duplex kidneys with ectopic ureters: ipsilateral ureteroureterostomy versus polar nephrectomy. J Urol. 1989; 142: 532-534.

3. Lais A, Peters CA: Laparoscopic Management of Duplication Anomalies. In: Docimo SG (ed.), Clinical Pediatric Urology. United Kingdom, Informa Healthcare. 2007, fifth edition, 649-54.

4. Parsons JK, Varkarakis I, Rha KH, Jarrett TW, Pinto PA, Kavoussi LR: Complications of abdominal urologic laparoscopy: longitudinal five-year analysis. Urology. 2004, 63: 27-32.

Dr. Henri Steyaert Pediatric Surgery and Urology Lenval Foundation for Children Nice, France E-mail: henri.steyaert@lenval.com 